Check for updates

Cite this: RSC Adv., 2019, 9, 92

Received 24th August 2018

Accepted 3rd December 2018

DOI: $10.1039 / c 8 r a 07074 a$

rsc.li/rsc-advances

\section{A new metallic $\pi$-conjugated carbon sheet used for the cathode of $\mathrm{Li}-\mathrm{S}$ batteries $\uparrow$}

\begin{abstract}
Li Zeng, (D) Yingxiang Cai, (D) * Zhihao Xiang, Yu Zhang and Xuechun Xu
Lithium-sulfur ( $\mathrm{Li}-\mathrm{S})$ batteries are considered as the most promising next generation high density energy storage devices. However, the commercialization of Li-S batteries is hindered by the shuttle effect of polysulfides, the low electronic conductivity of the sulfur cathode and a large volume expansion during lithiation. Herein, we predict a new two dimensional $\mathrm{sp}^{2}$ hybridized carbon allotrope (PHE-graphene) and prove its thermodynamic and kinetic stability. If it is utilized to encapsulate the cathode of Li-S batteries, not only will the shuttle effect be avoided but also the electronic conductivity of the sulfur cathode will be improved significantly owing to its metallic electronic band structure. The thermal conductivity of $\mathrm{PHE}$-graphene was found to be very high and even comparable with graphene, which is helpful for the heat dissipation of cathodes. In addition, PHE-graphene also exhibited superior mechanical properties including ideal tensile strength and in-plane stiffness.
\end{abstract}

\section{Introduction}

With the continuous consumption of finite fossil fuels and serious environmental pollution issues, the demand for renewable and clean energy sources such as solar and wind has increased substantially. Correspondingly, it is necessary to develop efficient rechargeable energy storage systems for these intermittent energy sources. ${ }^{1,2}$ Rechargeable lithium-ion (Liion) batteries are regarded as a suitable choice for energy storage devices. ${ }^{3,4}$ However, Li-ion batteries can still not meet the rising demands of large scale applications owing to their limited energy density and high cost. $^{3-5}$ Accordingly, new economical energy storage systems with a high specific capacity are needed. Li-air and $\mathrm{Li}-\mathrm{S}$ batteries have attracted increasing attention due to their high theoretical capacities of $3000 \mathrm{~W} \mathrm{~h} \mathrm{~kg}^{-1}$ and $2600 \mathrm{~W} \mathrm{~h} \mathrm{~kg}^{-1}$, respectively. ${ }^{1,6}$ Unfortunately, there are many insurmountable obstacles existing in $\mathrm{Li}^{-}$ air batteries such as a low practical energy density, ${ }^{6}$ lots of detrimental side reactions, ${ }^{7}$ instability towards moisture, ${ }^{8}$ etc. In contrast, it is more practical to deal with the challenges of $\mathrm{Li}-$ $\mathrm{S}$ batteries, and sulfur is environmentally friendly and abundant in nature. ${ }^{9}$ Therefore, Li-S batteries are widely viewed as promising energy storage systems to replace the present Li-ion batteries. Nevertheless, there are still a few challenges faced with $\mathrm{Li}-\mathrm{S}$ batteries. Firstly, sulfur and its reaction products $\left(\mathrm{Li}_{2} \mathrm{~S}\right.$ and $\mathrm{Li}_{2} \mathrm{~S}_{2}$ ) exhibit poor electronic conductivity, which results in a low rate capability and poor cycle performance of $\mathrm{Li}-\mathrm{S}$ batteries. ${ }^{10-12}$ Secondly, the dissolved intermediate lithium

Department of Physics, Nanchang University, Jiangxi, Nanchang 330031, P. R. China $\dagger$ Electronic supplementary information (ESI) available. See DOI: 10.1039/c8ra07074a polysulfides $\left(\mathrm{Li}_{2} \mathrm{~S}_{n}, 3 \leq n \leq 8\right)$ can diffuse freely between the cathode and anode during charge/discharge processes. This "shuttle effect" results in lithium anode corrosion, a poor cycle stability of the sulfur cathode, and low coulombic efficiency in Li-S batteries. ${ }^{13-15}$ Thirdly, the large volumetric expansion of the sulfur cathode during lithiation leads to electrode pulverization and fast capacity fading. ${ }^{16-18}$

To solve these problems faced with Li-S batteries, finding new cathode materials which not only have high electrical conductivity but also are able to confine polysulfides in the cathodes are crucial. Various sulfur-based composites have been proposed, such as carbon-sulfur composites, ${ }^{19-22}$ polymer-sulfur composites, ${ }^{23-25}$ and carbon-polymer-sulfur composites. ${ }^{26-28}$ Among the numerous available cathode materials, graphene has high interest due to its advantageous intrinsic properties for $\mathrm{Li}-\mathrm{S}$ batteries, such as superior electrical conductivity and high theoretical surface area for sulfur loading. ${ }^{29-31}$ However, graphene-sulfur composites have failed to eliminate the shuttle effect in Li-S batteries. ${ }^{32,33}$ To confine polysulfides effectively, methods using graphene oxide to replace graphene ${ }^{34}$ and encapsulating graphene-sulfur composites with polymers ${ }^{30}$ have been tried. However, both approaches will deteriorate the electrical conductivity of cathodes. ${ }^{32}$ Metal oxide-sulfur composites can also alleviate the shuttle effect by transforming soluble polysulfides into insoluble lower-order sulfides, ${ }^{35-37}$ but the poor electronic conductivity of these metal oxides limits their behavior for improving cell lifetimes. ${ }^{38} \mathrm{~A}$ recent study showed that two dimensional (2D) multiporous carbon sheets can be used to encapsulate the cathodes of Li-S batteries. ${ }^{39}$ Relative to sulfur-based composites, this multiporous carbon has a higher electrical conductivity. For new cathode materials, excellent mechanical 
properties are essential to bear possible volumetric expansion. In addition, high thermal conductivities are also expected for new cathode materials in order to facilitate the heat dissipation of electrodes.

In this study, we firstly predicted a new $2 \mathrm{D} \mathrm{sp}^{2}$ hybridized carbon allotrope (PHE-graphene), and suggested its synthesis by a dehydrogenation reaction of small molecules. Secondly, its thermodynamic and kinetic stability were examined and electronic band structures were investigated. Thirdly, the phonon lifetimes and group velocity in PHE-graphene were calculated. The phonon and electron thermal conductivities were evaluated, then the ideal tensile strength of PHE-graphene was determined. The upper limits of in-plane stiffness were systematically investigated for twenty $2 \mathrm{D}$ carbon sheets. Finally, the energy barriers of $\mathrm{Li}$ and $\mathrm{S}$ atoms diffusing through PHEgraphene were studied and its potential applications in $\mathrm{Li}-\mathrm{S}$ batteries are discussed.

\section{Methods}

Our $a b$ initio calculations were performed using the PerdewBurke-Ernzerhof (PBE) generalized gradient approximation ${ }^{\mathbf{4 0}}$ and implemented in the VASP code. ${ }^{\mathbf{4 1 4 2}}$ Blöchl's projector augmented wave (PAW) method ${ }^{\mathbf{4 3 , 4 4}}$ was utilized to describe the interactions between the nucleus and the valence electrons of carbon. A plane-wave basis with a cutoff energy of $400 \mathrm{eV}$ was used to expand the wave functions. The geometries of all of the carbon allotropes investigated in this work were fully relaxed including the atomic positions and lattice parameters until the residual forces on each atom were less than $0.0001 \mathrm{eV} \AA^{-1}$. The Brillouin zone sample meshes were dense enough and the $k$ spacing was less than $0.3 \AA^{-1}$ for all of the structures. The phonon band structure was determined by the direct supercell method as implemented in the Phonopy program. ${ }^{45}$ Lattice thermal conductivity was calculated by solving the linearized phonon Boltzmann equation within the single-mode relaxation time approximation (RTA) and implemented by the Phono3py code. ${ }^{46}$ Electron thermal conductivity was calculated according to the rigid-band approximation (RBA) using BoltzTraP2 code. ${ }^{47}$ The energy barriers of $\mathrm{S}$ and $\mathrm{Li}$ atoms diffusing were investigated using both PBE and dispersion corrected (DFT-D3) ${ }^{48}$ density functionals.

\section{Results and discussion}

Many methods can be utilized to prepare graphene, such as topdown exfoliation, bottom-up chemical vapor deposition (CVD) or chemical synthesis methods. ${ }^{49}$ However, new 2D carbon materials usually cannot be obtained by the exfoliation method due to the lack of bulk layer precursors. CVD is also not suitable for the synthesis of 2D carbon except for graphene. Chemical synthesis from small molecules is a promising route to prepare new 2D carbon sheets, although it is still very challenging. Experimentally, two 2D carbon sheets have been synthesized by the dehydration or dehydrogenation chemical reactions of small molecules as shown in Fig. 1a and b. A 4-6 carbophene (i.e. DHP-graphene $)^{\mathbf{3 9 , 5 0}}$ was synthesized by the dehydration

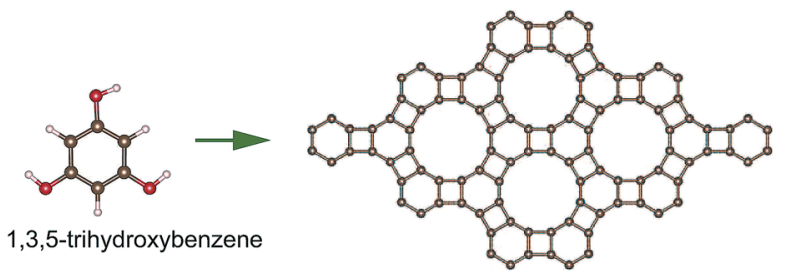

(a)
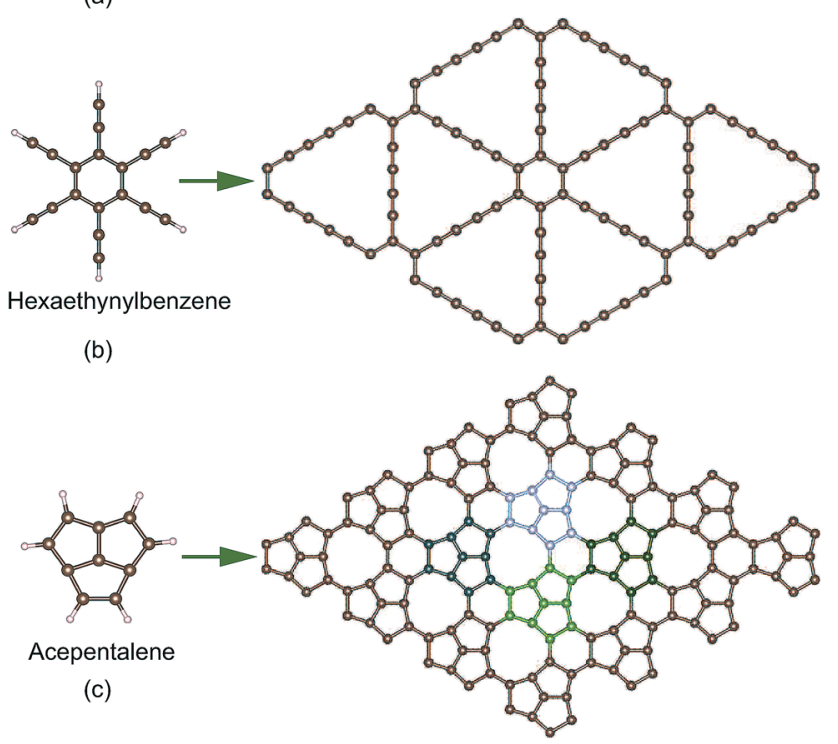

Fig. 1 (a) The 4-6 carbophene (i.e. DHP-graphene) prepared by the dehydration reaction of 1,3,5-trihydroxybenzene molecules. (b) Graphdiyne synthesized by the dehydrogenation reaction of hexaethynylbenzene molecules. (c) A possible preparation method for $\mathrm{PHE}-$ graphene by the dehydrogenation reaction of acepentalene molecules.

reaction of 1,3,5-trihydroxybenzene molecules and graphdiyne ${ }^{51}$ was obtained by the dehydrogenation reaction of hexaethynylbenzene molecules. Likewise, a new conjugated $\pi$ bonding planar carbon structure might be achieved by the dehydrogenation reaction of acepentalene ${ }^{52}$ molecules as shown in Fig. 1c. This carbon allotrope has also been found in structure research based on particle swarm optimization for a system with ten carbon atoms. Its structure consists of pentagonal, hexagonal and enneagonal rings, and thus it is named PHE-graphene. PHE-graphene belongs to the space group $P \overline{6} m 2\left(D_{3 \mathrm{~h}}\right)$. Its lattice constant $a$ is $5.738 \AA$. In the unit cell of PHE-graphene, there are four nonequivalent chemical bonds, whose lengths are 1.385, 1.448, 1.392 and $1.514 \AA$, respectively. The ratio of chemical bond numbers is $1: 2: 1: 1$. Thus the average bond length is $1.438 \AA$ which is a little higher than that of graphene (1.42 $\AA$ ).

To add PHE-graphene into the carbon material family, we should firstly check its thermodynamic and kinetic stability. Fig. 2a shows the total energies of PHE-graphene and nineteen other 2D carbon allotropes, including DHP-graphene, ${ }^{39}$ graphdiyne, ${ }^{51}$ THD-graphene, ${ }^{53}$ planar T-graphene, ${ }^{54}$ rectangular heackelite, ${ }^{55}$ pza-C $_{10},{ }^{56}$ phagraphene, ${ }^{57}$ pentahexoctite,${ }^{58}$ pentaheptite, ${ }^{59}$ OPG-L ${ }^{60}$ oblique heackelite, ${ }^{55}$ HOP graphene, ${ }^{61}$ hexagonal heackelite, ${ }^{55}$ graphene, ${ }^{62} \quad \gamma$-graphyne, ${ }^{63}$ 


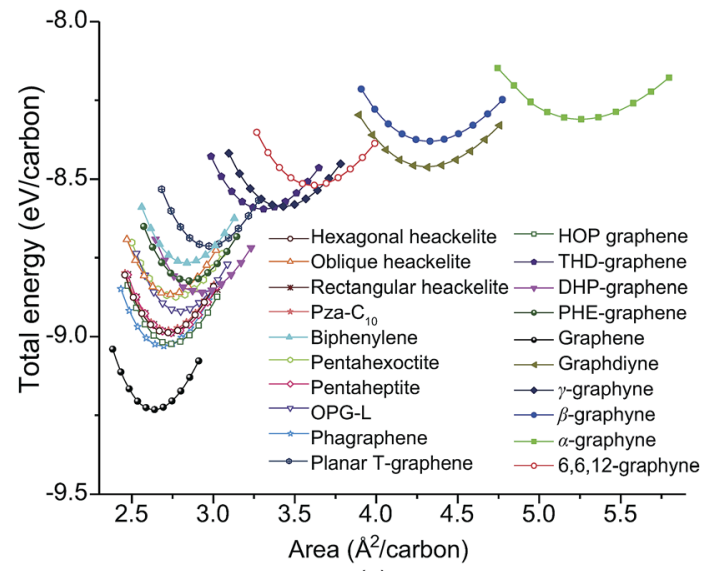

(a)

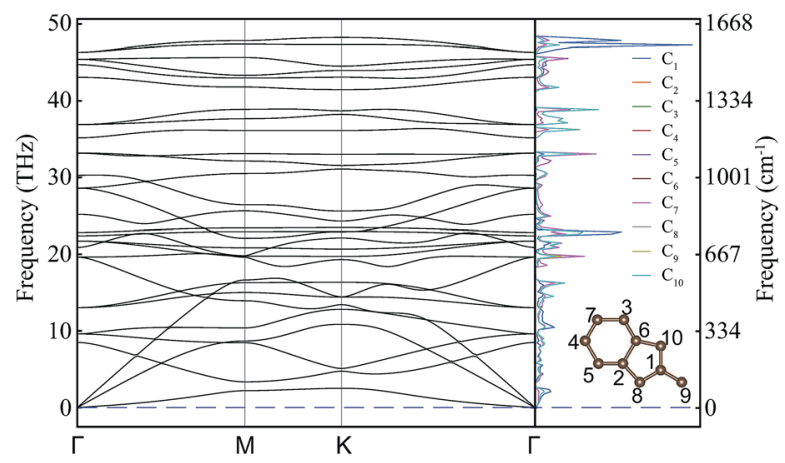

(b)

Fig. 2 (a) Total energy as a function of volume for the 2D carbon allotropes. (b) Phonon dispersion of PHE-graphene and projected phonon density of states (PhDOS). The insert shows the ten nonequivalent carbon atoms in the unit cell.

biphenylene, ${ }^{64} \beta$-graphyne, ${ }^{65} \alpha$-graphyne ${ }^{65}$ and 6,6,12-graphyne. ${ }^{65}$ As expected, graphene is the most stable $2 \mathrm{D}$ carbon layer. Five $\mathrm{sp}+\mathrm{sp}^{2}$ hybridized structures (including $\alpha$-graphyne, $\beta$ graphyne, $\gamma$-graphyne, 6,6,12-graphyne and graphdiyne) were less stable relative to the other $\mathrm{sp}^{2}$ hybridized carbon sheets. Among them, the $\alpha$-graphyne is the least stable $\mathrm{sp}+\mathrm{sp}^{2}$ hybridized structure and graphdiyne is the least stable structure experimentally synthesized. PHE-graphene also exhibited a high thermodynamic stability. At equilibrium geometry, its total energy was lower than not only the $\mathrm{sp}+\mathrm{sp}^{2}$ hybridized 2D carbon structures but also $\mathrm{sp}^{2}$ hybridized biphenylene, planar T-graphene and THD-graphene. Fig. $2 \mathrm{~b}$ shows the phonon dispersion and projected phonon density of states (PhDOS) of PHE-graphene. Since no imaginary phonon frequencies were found, PHE-graphene is kinetically stable. The highest phonon frequency of PHE-graphene was calculated to be $48.24 \mathrm{THz}$ (or $1609 \mathrm{~cm}^{-1}$ ), which is extremely close to that (around $1600 \mathrm{~cm}^{-1}$ (ref. 66 and 67)) of graphite and graphene. In addition, we could find two phonon bandgaps in the frequency regions of 33-35 and 39-42 THz. From the PhDOS, it can be seen that the high frequency phonons were mainly from the contribution of the $\mathrm{C}_{1}$ atom. Furthermore, a NVT ab initio molecular dynamics simulation was performed, which also confirmed the stability of PHE-graphene (see Fig. S1 in the ESI†े).
PHE-graphene has ten carbon atoms and forty $2 \mathrm{~s}^{2} 2 \mathrm{p}^{2}$ electrons in its unit cell. If PHE-graphene is semiconductive, the $20^{\text {th }}$ band should be below the Fermi level $\left(E_{\mathrm{F}}\right)$ and the $21^{\text {st }}$ band should be above the $E_{\mathrm{F}}$. From Fig. 3a, we can find that both the $20^{\text {th }}$ and $21^{\text {st }}$ bands were partially occupied and crossed the $E_{\mathrm{F}}$. Therefore, PHE-graphene is a metallic carbon material. Fig. $3 \mathrm{~b}$ shows the projected density of states (PDOS: $\mathrm{s}, \mathrm{p}_{x}, \mathrm{p}_{y}$ and $\mathrm{p}_{z}$ ). The PDOS near $E_{\mathrm{F}}$ completely results from the $\mathrm{p}_{z}$ electrons, which discloses the $\pi$-conjugated nature of PHE-graphene. If the $\mathrm{p}_{z}$ DOS is projected on each carbon atom, it can be seen that the PDOS near the $E_{\mathrm{F}}$ is mainly ascribed to the contributions of the $\mathrm{C}_{8}, \mathrm{C}_{9}$ and $\mathrm{C}_{10}$ atoms as shown in Fig. 3c. Based on the DOS analysis, we can conclude that PHE-graphene is a metallic $\pi$ bonding carbon structure with excellent electrical conductivity. Fig. 3d shows the charge densities of the $20^{\text {th }}$ and $21^{\text {st }}$ bands. It can be seen that the electrons in PHE-graphene are delocalized especially for the $20^{\text {th }}$ band, in which the electrons behave just like the free electrons in metals.

The thermal conductivity of a material results from the transportation of electrons and phonons. Fig. 4a shows the phonon lifetimes calculated from the imaginary part of phonon self energy ${ }^{46}$ We find that most phonons have short lifetimes, especially the high frequency phonons. The longest phonon lifetime around $20 \mathrm{THz}$ is still less than 7 ps. Fig. $4 \mathrm{~b}$ shows the phonon group velocity. Only a few low frequency phonons have group velocities of more than $10 \mathrm{~km} \mathrm{~s}^{-1}$ and most phonons' group velocities are less than $5 \mathrm{~km} \mathrm{~s}^{-1}$. Two phonon bandgaps around $33-35$ and $39-42 \mathrm{THz}$ can also be found from Fig. $4 \mathrm{a}$ and

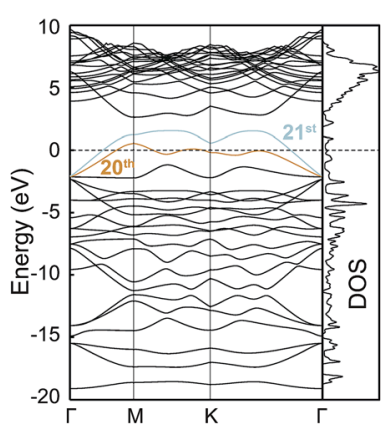

(a)

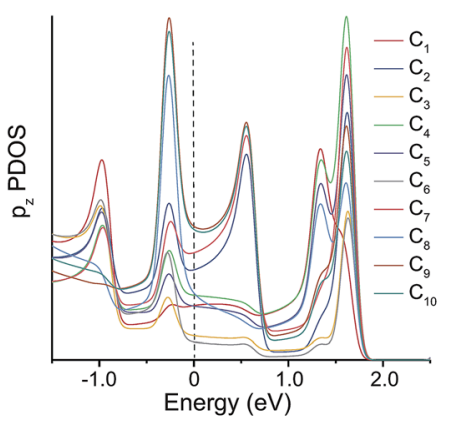

(c)

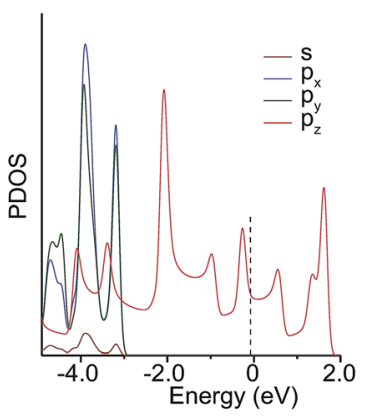

(b)

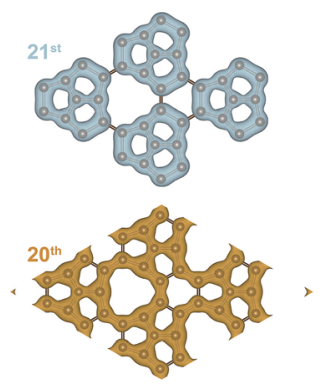

(d)
Fig. 3 (a) Band structures and the density of states (DOS) of PHEgraphene. (b) Partial density of states (PDOS) of $s, p_{x}, p_{y}$ and $p_{z}$. (c) The $p_{z}$ PDOS of the $C_{1}-C_{10}$ atoms. (d) Charge densities of the $20^{\text {th }}$ and $21^{\text {st }}$ bands. 


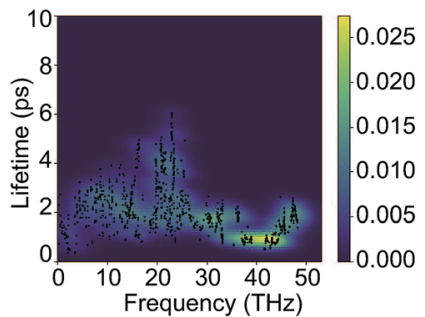

(a)

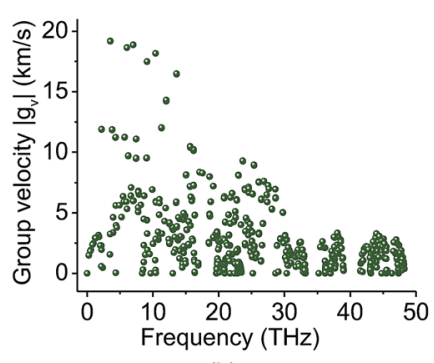

(b)

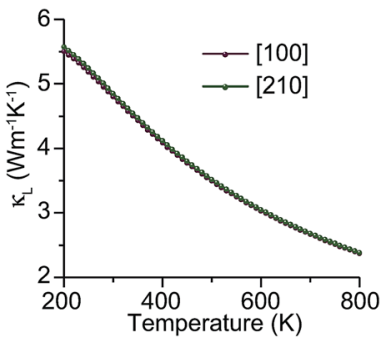

(c)

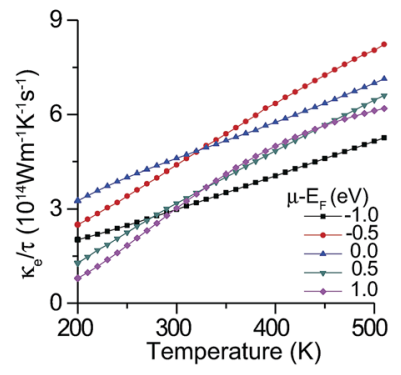

(d)
Fig. 4 (a) Phonon lifetimes. (b) Phonon group velocity. (c) Lattice thermal conductivity $\left(\kappa_{\mathrm{L}}\right)$ of PHE-graphene as a function of temperature. (d) Electron thermal conductivity $\left(\kappa_{\mathrm{e}}\right)$ divided by relaxation time $(\tau)$ as a function of temperature.

b. Due to low phonon lifetimes and group velocity, the lattice thermal conductivity $\left(\kappa_{\mathrm{L}}\right)$ of PHE-graphene is quite low. At room temperature $(300 \mathrm{~K})$, the $\kappa_{\mathrm{L}}$ is only $4.8 \mathrm{~W} \mathrm{~m}^{-1} \mathrm{~K}^{-1}$. Along the [100] and [210] directions, thermal transport is almost isotropic as shown in Fig. 4c. With increasing temperature, $\kappa_{\mathrm{L}}$ decreases drastically due to the enhancement of phonon dispersion. The electron thermal conductivity $\left(\kappa_{\mathrm{e}}\right)$ is the function of both temperature and chemical potential $(\mu)$. Owing to the metallic electronic band structure, $\kappa_{\mathrm{e}}$ should dominate the thermal transport of PHE-graphene just like other metals. Fig. $4 \mathrm{~d}$ shows $\kappa_{\mathrm{e}} / \tau$ as functions of temperature for five $\mu-E_{\mathrm{F}}$, where $\mu$ is chemical potential. It can be seen that $\kappa_{\mathrm{e}} / \tau$ increases with rising temperature. While $\mu-E_{\mathrm{F}}$ is $-1 \mathrm{eV}, \kappa_{\mathrm{e}} / \tau$ is $2.986 \times$ $10^{14} \mathrm{~W} \mathrm{~m}^{-1} \mathrm{~K}^{-1} \mathrm{~s}^{-1}$. Accurate electron relaxation time of PHEgraphene is not available at present. However, a few studies of 2D carbon materials have confirmed that $\tau$ is usually tens of picoseconds (ps). For instance, $\tau$ is $14.6 \mathrm{ps}$ for THD-graphene, ${ }^{53}$ 13-14 ps for graphene $e^{68}$ and $16-19$ ps for graphdiyne. ${ }^{69}$ Supposing the $\tau$ of PHE-graphene is $14 \mathrm{ps}$, close to $\mathrm{sp}^{2}$ hybridized THD-graphene and graphene, $\kappa_{\mathrm{e}}$ is estimated to be up to $4180 \mathrm{~W} \mathrm{~m}^{-1} \mathrm{~K}^{-1}$ while $\mu-E_{\mathrm{F}}$ is $-1.0 \mathrm{eV}$. Such a high thermal conductivity of PHE-graphene is even comparable with singlelayer graphene $\left(5300 \mathrm{~W} \mathrm{~m}^{-1} \mathrm{~K}^{-1}\right),{ }^{70}$ and superior to few-layer graphene $\left(1300-2800 \quad \mathrm{~W} \quad \mathrm{~m}^{-1} \quad \mathrm{~K}^{-1}\right),^{71}$ bulk graphite $\left(2000 \mathrm{~W} \mathrm{~m}^{-1} \mathrm{~K}^{-1}\right){ }^{72}$ carbon nanotubes $\left(3000 \mathrm{~W} \mathrm{~m}^{-1} \mathrm{~K}^{-1}\right)^{73}$ and diamond $\left(1000-2200 \mathrm{~W} \mathrm{~m}^{-1} \mathrm{~K}^{-1}\right) \cdot^{74}$

Fig. 5a shows the stress-strain relations. We found that the ideal tensile strength of PHE-graphene is up to $71.6 \mathrm{GPa}$ which is far less than that $(130 \pm 10 \mathrm{GPa})$ of graphene. ${ }^{75}$ However, PHE-graphene still has a higher tensile strength relative to most 2D materials, such as $\operatorname{MoS}_{2}(25 \mathrm{GPa}){ }^{76}$ silicene $(12.5 \mathrm{GPa})^{77}$ and phosphorene (4.45 GPa). ${ }^{78}$ Before yielding, PHE-graphene can endure $15 \%$ tensile strain. As for 2D PHE-graphene, its elastic behavior under strain should be evaluated by in-plane stiffness $C$ rather than Young's modulus. If $S_{0}$ is defined as the area of PHE-graphene at equilibrium geometry, the in-plane stiffness can be given by $C=\left(1 / S_{0}\right) \times\left(\partial^{2} E / \partial \varepsilon^{2}\right)$, where $E$ is the strain energy calculated by subtracting the total energy of the strained system from the equilibrium total energy and $\varepsilon$ is the strain. In this study, we applied strain $\varepsilon$ on both the [100] and [010] directions at the same time, which meant that the $2 \mathrm{D}$ lattice constants or the unit cell were enlarged $(1+\varepsilon)$ times. Thus, we calculated the upper limit of the stiffness, i.e. $C_{\max }$. Fig. $5 \mathrm{~b}$ shows the $C_{\max }$ of the twenty carbon allotropes. It can be found that the $C_{\max }$ significantly depends on the number of carbon atoms per unit area, $N_{\sigma}$. For $\mathrm{sp}+\mathrm{sp}^{2}$ hybridized carbon sheets ( $\alpha$-graphyne, $\beta$-graphyne, graphdiyne, 6,6,12-graphyne, and $\gamma$ graphyne), they usually have a lower $C_{\max }$ due to their small $N_{\sigma}$. In contrast, $C_{\max }$ is usually higher than $300 \mathrm{~N} \mathrm{~m}^{-1}$ for $\mathrm{sp}^{2}$ hybridized carbon allotropes except for THD-graphene. Graphene has the highest $N_{\sigma}\left(0.379 \AA^{-1}\right)$ and its $C_{\max }$ is up to $422 \mathrm{~N}$ $\mathrm{m}^{-1}$, which is very close to the experimental upper limit (about $419 \mathrm{~N} \mathrm{~m}^{-1}$ ) of graphene. ${ }^{75}$ The $C_{\max }$ of PHE-graphene was also up to $353 \mathrm{~N} \mathrm{~m}^{-1}$ and thus it possesses excellent mechanical properties.

Fig. 6a shows the energy barrier of sulfur atoms passing through the enneagonal rings of PHE-graphene. It can be seen that a very high barrier prevents the sulfur atoms from penetrating through PHE-graphene. When one sulfur atom gradually approaches PHE-graphene, the total energy drops by $0.5 \mathrm{eV}$. This indicates that PHE-graphene has weak adsorption for sulfur atoms. The effects of the PBE functional and the DFT-D3 dispersion corrected functional on energy barriers are negligible. Surprisingly, we find that PHE-graphene strongly adsorbs $\mathrm{Li}$ atoms as shown in Fig. $6 \mathrm{~b}$. For the most stable adsorption geometry, the distance between Li and PHE-graphene was about $1.08 \AA$ and the adsorption energy was 1.97 (1.90) eV at the PBE (DFT-D3) level. If one $\mathrm{Li}$ atom passes through the PHEgraphene, only an energy barrier around $0.47-0.48 \mathrm{eV}$ needs to be overcome. In view of PHE-graphene preventing sulfur passing through it, PHE-graphene could be used to encapsulate the cathodes of Li-S batteries, by which the "shuttle effect" could be effectively avoided. The adsorption geometries and energies of $\mathrm{Li}_{2} \mathrm{~S}_{n}(n \leq 8)$ and $\mathrm{S}_{8}$ on PHE-graphene were also evaluated as shown in Table. S1 and Fig. S2 (see the ESI $\dagger$ ). The adsorption energy was the highest for $\mathrm{Li}_{2} \mathrm{~S}$ but was relatively weak for $\operatorname{Li}_{2} \mathrm{~S}_{n}(3 \leq n \leq 8)$ and $\mathrm{S}_{8}$ species. In addition, the low conductivity faced in Li-S batteries at present can also be significantly improved because PHE-graphene is a metallic carbon material. Fig. $6 \mathrm{c}$ shows the vertical distance $(\Delta d)$ between Li and PHE-graphene during Li atom diffusion on the PHE-graphene surface. The minimum $\Delta d$ occurred at the enneagonal hollow sites and was around $1.08 \AA$ A. In contrast, the $\Delta d$ was at a maximum on the top of $\mathrm{C}$, which was about $1.97 \AA$. The Li atom diffusion barriers on the PHE-graphene surface are presented in Fig. 6d. The diffusion barriers between adjacent carbon rings were $0.589,0.548,0.301$, and $0.302 \mathrm{eV}$ for enneagon $\rightarrow$ hexagon, enneagon $\rightarrow$ pentagon, hexagon $\rightarrow$ pentagon, 


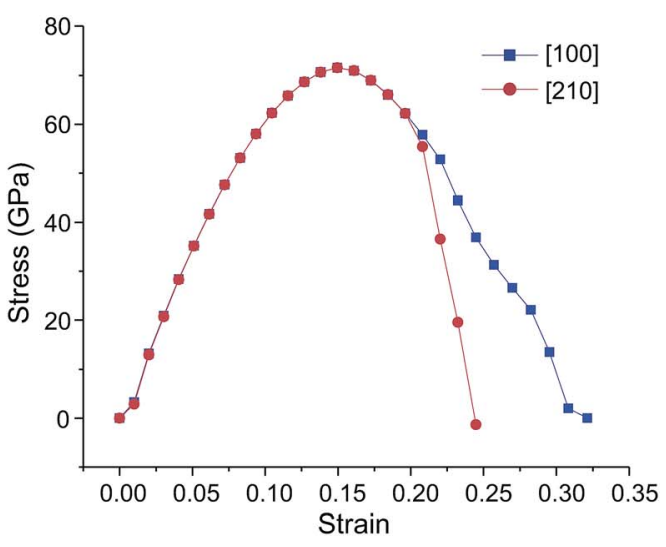

(a)

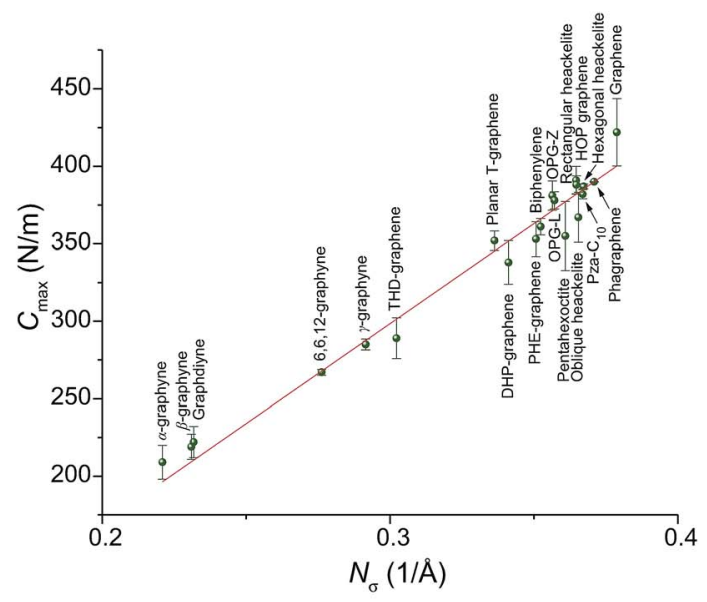

(b)

Fig. 5 (a) The stress-strain relations of PHE-graphene in the [100] and [210] directions. (b) The upper limit of in-plane stiffness $\left(C_{\text {max }}\right)$ as a function of the number $\left(N_{\sigma}\right)$ of carbon atoms per unit area.

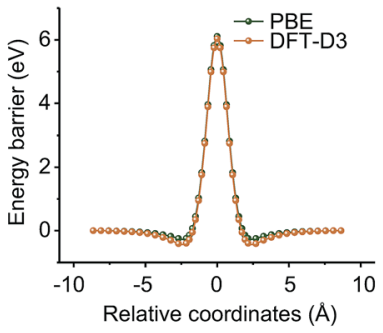

(a)

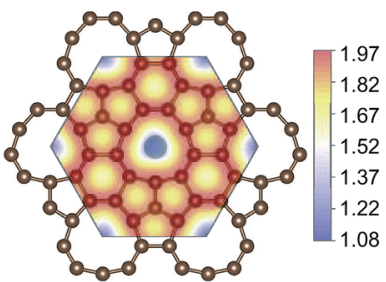

(c)

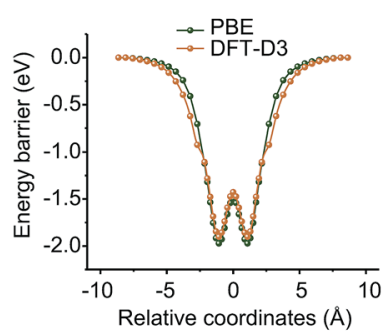

(b)

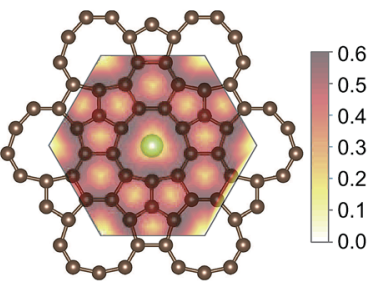

(d)
Fig. 6 (a) and (b) show the energy barriers of $S$ and Li atoms diffusing through the enneagon rings, respectively. (c) The vertical distance $(\Delta d)$ between $\mathrm{Li}$ and the PHE-graphene during $\mathrm{Li}$ atom diffusion on the surface of PHE-graphene. (d) A 2D contour plot of the energy barrier for $\mathrm{Li}$ atom diffusion on the surface of PHE-graphene.

and pentagon $\rightarrow$ pentagon, and $0.395,0.339,0.286$, and $0.302 \mathrm{eV}$ for the reverse processes, respectively. Therefore, Li atoms shift readily along the path of $\cdots$ hexagon $\rightarrow$ pentagon $\rightarrow$ pentagon $\rightarrow$ hexagon $\cdots$ and the corresponding barriers were close to that $(0.327 \mathrm{eV})$ of $\mathrm{Li}$ atom diffusion on the graphene surface. ${ }^{79}$

\section{Conclusions}

In summary, we proposed a new carbon sheet (PHE-graphene) and suggested its preparation by a dehydrogenation reaction of small molecules. The thermodynamic and kinetic stability of
PHE-graphene were examined by means of ab initio calculations. Electronic band structure calculations showed that PHEgraphene is a metallic carbon material. The density of states near the Fermi energy level completely result from the contributions of delocalized $\mathrm{p}_{z}$ electrons. Similar to graphene, PHEgraphene is a typical $\pi$-conjugated system. Anharmonic lattice dynamics calculations revealed the low phonon lifetimes and group velocity in PHE-graphene, which directly results in its low lattice thermal conductivity. However, the electron thermal conductivity was found to be very high and even comparable with graphene. The enneagonal rings in PHE-graphene provide a passage for $\mathrm{Li}$ atoms but prevent $\mathrm{S}$ atoms from passing through. If PHE-graphene is utilized in cathodes, the major challenges faced by Li-S batteries, including low conductivity and the shuttle effect can be solved. Its high thermal conductivity is also helpful for the heat dissipation of cathodes. In addition, PHE-graphene also exhibits superior mechanical properties. Its ideal tensile strength was up to $71.6 \mathrm{GPa}$ and the upper limit of its in-plane stiffness was $353 \mathrm{~N} \mathrm{~m}^{-1}$.

\section{Conflicts of interest}

There are no conflicts to declare.

\section{Acknowledgements}

This work was supported financially by the National Natural Science Foundation of China (Grant No. 11464028), the Natural Science Foundation of Jiangxi Province, China (Grant No. 20171ACB21007) and the Department of Education of Jiangxi Province, China (Grant No. GJJ150025).

\section{Notes and references}

1 M. Arumugam, C. Sheng-Heng and Z. Chenxi, Adv. Mater., 2015, 27, 1980-2006. 
2 J.-Q. Huang, T.-Z. Zhuang, Q. Zhang, H.-J. Peng, C.-M. Chen and F. Wei, ACS Nano, 2015, 9, 3002-3011.

3 X. Yu, Z. Bi, F. Zhao and A. Manthiram, ACS Appl. Mater. Interfaces, 2015, 7, 16625-16631.

4 A. Manthiram, Y. Fu and Y.-S. Su, Acc. Chem. Res., 2013, 46, 1125-1134.

5 J. Xiao, J. Z. Hu, H. Chen, M. Vijayakumar, J. Zheng, H. Pan, E. D. Walter, M. Hu, X. Deng, J. Feng, B. Y. Liaw, M. Gu, Z. D. Deng, D. Lu, S. Xu, C. Wang and J. Liu, Nano Lett., 2015, 15, 3309-3316.

6 A. Kraytsberg and Y. Ein-Eli, J. Power Sources, 2011, 196, 886893.

7 L. Li, S.-H. Chai, S. Dai and A. Manthiram, Energy Environ. Sci., 2014, 7, 2630-2636.

8 L. Jang-Soo, T. K. Sun, C. Ruiguo, C. Nam-Soon, L. Meilin, L. K. Tae and C. Jaephil, Adv. Energy Mater., 2010, 1, 34-50.

9 Q. Wang, N. Yan, M. Wang, C. Qu, X. Yang, H. Zhang, X. Li and H. Zhang, ACS Appl. Mater. Interfaces, 2015, 7, 2500225006.

10 D. Bing, Y. Changzhou, S. Laifa, X. Guiyin, N. Ping and Z. Xiaogang, Chem.-Eur. J., 2013, 19, 1013-1019.

11 P. G. Bruce, S. A. Freunberger, L. J. Hardwick and J.-M. Tarascon, Nat. Mater., 2011, 11, 19.

12 X. Ji and L. F. Nazar, J. Mater. Chem., 2010, 20, 9821-9826.

13 B. Zhang, X. Qin, G. R. Li and X. P. Gao, Energy Environ. Sci., 2010, 3, 1531-1537.

14 K. Kumaresan, Y. Mikhaylik and R. E. White, J. Electrochem. Soc., 2008, 155, A576-A582.

15 Y. V. Mikhaylik and J. R. Akridge, J. Electrochem. Soc., 2004, 151, A1969-A1976.

16 Y. Wei, Y. Tao, C. Zhang, J. Wang, W. Qiao, L. Ling and D. Long, Electrochim. Acta, 2016, 188, 385-392.

17 G. Zheng, Y. Yang, J. J. Cha, S. S. Hong and Y. Cui, Nano Lett., 2011, 11, 4462-4467.

18 J. Zang, T. An, Y. Dong, X. Fang, M. Zheng, Q. Dong and N. Zheng, Nano Res., 2015, 8, 2663-2675.

19 H. Wang, Y. Yang, Y. Liang, J. T. Robinson, Y. Li, A. Jackson, Y. Cui and H. Dai, Nano Lett., 2011, 11, 2644-2647.

20 X. Ji, K. T. Lee and L. F. Nazar, Nat. Mater., 2009, 8, 500.

21 X. Li, Y. Cao, W. Qi, L. V. Saraf, J. Xiao, Z. Nie, J. Mietek, J.-G. Zhang, B. Schwenzer and J. Liu, J. Mater. Chem., 2011, 21, 16603-16610.

22 S. Wei, H. Zhang, Y. Huang, W. Wang, Y. Xia and Z. Yu, Energy Environ. Sci., 2011, 4, 736-740.

23 Y. Fu and A. Manthiram, J. Phys. Chem. C, 2012, 116, 89108915.

24 Y. Zhang, Z. Bakenov, Y. Zhao, A. Konarov, T. N. L. Doan, M. Malik, T. Paron and P. Chen, J. Power Sources, 2012, 208, 1-8.

25 L. Xiao, Y. Cao, J. Xiao, B. Schwenzer, M. H. Engelhard, L. V. Saraf, Z. Nie, G. J. Exarhos and J. Liu, Adv. Mater., 2012, 24, 1176-1181.

26 Y. Bu, J. Wu, X. Zhao, K. Ding, Q. Liu, Y. Huang, J. Lv and Y. Wang, RSC Adv., 2016, 6, 104591-104596.

27 G. Zheng, Q. Zhang, J. J. Cha, Y. Yang, W. Li, Z. W. Seh and Y. Cui, Nano Lett., 2013, 13, 1265-1270.
28 X. Li, M. Rao and W. Li, J. Solid State Electrochem., 2016, 20, 153-161.

29 G. Zhou, L.-C. Yin, D.-W. Wang, L. Li, S. Pei, I. R. Gentle, F. Li and H.-M. Cheng, ACS Nano, 2013, 7, 5367-5375.

30 Z. Chenxi and M. Arumugam, Adv. Energy Mater., 2013, 3, 1008-1012.

31 T. Lin, Y. Tang, Y. Wang, H. Bi, Z. Liu, F. Huang, X. Xie and M. Jiang, Energy Environ. Sci., 2013, 6, 1283-1290.

32 X. Yang, L. Zhang, F. Zhang, Y. Huang and Y. Chen, ACS Nano, 2014, 8, 5208-5215.

33 Z. Li, Y. Huang, L. Yuan, Z. Hao and Y. Huang, Carbon, 2015, 92, 41-63.

34 L. Ji, M. Rao, H. Zheng, L. Zhang, Y. Li, W. Duan, J. Guo, E. J. Cairns and Y. Zhang, J. Am. Chem. Soc., 2011, 133, 18522-18525.

35 Z. Wei Seh, W. Li, J. J. Cha, G. Zheng, Y. Yang, M. T. Mcdowell, P.-C. Hsu and Y. Cui, Nat. Commun., 2013, 4, 1331.

36 X. Tao, J. Wang, Z. Ying, Q. Cai, G. Zheng, Y. Gan, H. Huang, Y. Xia, C. Liang, W. Zhang and Y. Cui, Nano Lett., 2014, 14, 5288-5294.

37 Q. Pang, D. Kundu, M. Cuisinier and L. F. Nazar, Nat. Commun., 2014, 5, 4759.

38 T. Zeng, X. Hu, P. Ji and G. Zhou, Solid State Ionics, 2016, 291, 47-68.

39 Y. Cai, Y. Guo, B. Jiang and Y. Lv, Sci. Rep., 2017, 7, 14948.

40 J. P. Perdew, K. Burke and M. Ernzerhof, Phys. Rev. Lett., 1996, 77, 3865-3868.

41 G. Kresse and J. Furthmüller, Comput. Mater. Sci., 1996, 6, 15-50.

42 G. Kresse and J. Furthmüller, Phys. Rev. B: Condens. Matter Mater. Phys., 1996, 54, 11169-11186.

43 P. E. Blöchl, Phys. Rev. B: Condens. Matter Mater. Phys., 1994, 50, 17953-17979.

44 G. Kresse and D. Joubert, Phys. Rev. B: Condens. Matter Mater. Phys., 1999, 59, 1758-1775.

45 A. Togo and I. Tanaka, Scr. Mater., 2015, 108, 1-5.

46 A. Togo, L. Chaput and I. Tanaka, Phys. Rev. B: Condens. Matter Mater. Phys., 2015, 91, 094306.

47 G. K. Madsen, J. Carrete and M. J. Verstraete, Comput. Phys. Commun., 2018, 231, 140-145.

48 S. Grimme, J. Antony, S. Ehrlich and H. Krieg, J. Chem. Phys., 2010, 132, 154104.

49 X. Cai, Y. Luo, B. Liu and H.-M. Cheng, Chem. Soc. Rev., 2018, 47, 6224-6266.

50 Q.-S. Du, P.-D. Tang, H.-L. Huang, F.-L. Du, K. Huang, N.-Z. Xie, S.-Y. Long, Y.-M. Li, J.-S. Qiu and R.-B. Huang, Sci. Rep., 2017, 7, 40796.

51 M. Long, L. Tang, D. Wang, Y. Li and Z. Shuai, ACS Nano, 2011, 5, 2593-2600.

52 A. de Meijere, P. R. Schreiner, F.-M. Schüngel, F. Gerson and P. Merstetter, Chem. Commun., 1999, 2189-2190.

53 X. Xu, B. Jiang, Y. Guo and Y. Cai, Mater. Chem. Phys., 2017, 200, 50-56.

54 H. Huang, Y. Li, Z. Liu, J. Wu and W. Duan, Phys. Rev. Lett., 2013, 110, 029603. 
55 H. Terrones, M. Terrones, E. Hernández, N. Grobert, J.-C. Charlier and P. M. Ajayan, Phys. Rev. Lett., 2000, 84, 1716-1719.

56 X. Luo, L.-M. Liu, Z. Hu, W.-H. Wang, W.-X. Song, F. Li, S.-J. Zhao, H. Liu, H.-T. Wang and Y. Tian, J. Phys. Chem. Lett., 2012, 3, 3373-3378.

57 Z. Wang, X.-F. Zhou, X. Zhang, Q. Zhu, H. Dong, M. Zhao and A. R. Oganov, Nano Lett., 2015, 15, 6182-6186.

58 B. R. Sharma, A. Manjanath and A. K. Singh, Sci. Rep., 2014, 4, 7164 .

59 V. H. Crespi, L. X. Benedict, M. L. Cohen and S. G. Louie, Phys. Rev. B: Condens. Matter Mater. Phys., 1996, 53, R13303-R13305.

60 C. Su, H. Jiang and J. Feng, Phys. Rev. B: Condens. Matter Mater. Phys., 2013, 87, 075453.

61 B. Mandal, S. Sarkar, A. Pramanik and P. Sarkar, Phys. Chem. Chem. Phys., 2013, 15, 21001-21006.

62 J. C. Meyer, A. K. Geim, M. I. Katsnelson, K. S. Novoselov, T. J. Booth and S. Roth, Nature, 2007, 446, 60.

63 N. Narita, S. Nagai, S. Suzuki and K. Nakao, Phys. Rev. B: Condens. Matter Mater. Phys., 1998, 58, 11009-11014.

64 M. A. Hudspeth, B. W. Whitman, V. Barone and J. E. Peralta, ACS Nano, 2010, 4, 4565-4570.

65 D. Malko, C. Neiss, F. Viñes and A. Görling, Phys. Rev. Lett., 2012, 108, 086804.

66 J. Maultzsch, S. Reich, C. Thomsen, H. Requardt and P. Ordejón, Phys. Rev. Lett., 2004, 92, 075501.
67 O. Dubay and G. Kresse, Phys. Rev. B: Condens. Matter Mater. Phys., 2003, 67, 035401.

68 J. Xi, M. Long, L. Tang, D. Wang and Z. Shuai, Nanoscale, 2012, 4, 4348-4369.

69 M. Long, L. Tang, D. Wang, Y. Li and Z. Shuai, ACS Nano, 2011, 5, 2593-2600.

70 A. A. Balandin, S. Ghosh, W. Bao, I. Calizo, D. Teweldebrhan, F. Miao and C. N. Lau, Nano Lett., 2008, 8, 902-907.

71 S. Ghosh, W. Bao, D. L. Nika, S. Subrina, E. P. Pokatilov, C. N. Lau and A. A. Balandin, Nat. Mater., 2010, 9, 555.

72 A. A. Balandin, Nat. Mater., 2011, 10, 569.

73 H.-Y. Chiu, V. V. Deshpande, H. W. C. Postma, C. N. Lau, C. Mikó, L. Forró and M. Bockrath, Phys. Rev. Lett., 2005, 95, 226101.

74 A. Sukhadolau, E. Ivakin, V. Ralchenko, A. Khomich, A. Vlasov and A. Popovich, Diamond Relat. Mater., 2005, 14, 589-593.

75 C. Lee, X. Wei, J. W. Kysar and J. Hone, Science, 2008, 321, 385-388.

76 T. Li, Phys. Rev. B: Condens. Matter Mater. Phys., 2012, 85, 235407.

77 Q.-X. Pei, Z.-D. Sha, Y.-Y. Zhang and Y.-W. Zhang, J. Appl. Phys., 2014, 115, 023519.

78 Z.-D. Sha, Q.-IX. Pei, Z. Ding, J.-W. Jiang and Y.-W. Zhang, J. Appl. Phys., 2015, 48, 395303.

79 C. Uthaisar and V. Barone, Nano Lett., 2010, 10, 2838-2842. 